\title{
Identification of DNA-Repair-Related
} Five-Gene Signature to Predict Prognosis in Patients with Esophageal Cancer

\section{OPEN ACCESS}

Edited by:

Andrea Ladányi,

National Institute of Oncology (NIO),

Hungary

${ }^{*}$ Correspondence:

Miao He

hemiao_cmu@126.com

Lin Zhao

Izhao@cmu.edu.cn

${ }^{\dagger}$ These authors have contributed equally to this work

Received: 20 August 2020 Accepted: 10 February 2021

Published: 30 March 2021

Citation:

Wang L, LiX, Zhao L, Jiang L, Song $X$, Qi A, Chen T, Ju M, Hu B, Wei M, He M and Zhao $L$ (2021) Identification of DNA-Repair-Related Five-Gene Signature to Predict Prognosis in

Patients with Esophageal Cancer. Pathol. Oncol. Res. 27:596899. doi: 10.3389/pore.2021.596899

\begin{abstract}
Lin Wang ${ }^{1,2 \dagger}$, Xueping $L^{1,2 \dagger}$, Lan Zhao ${ }^{1,2}$, Longyang Jiang ${ }^{1,2}$, Xinyue Song ${ }^{1,2}$, Aoshuang Qi ${ }^{1,2}$, Ting Chen ${ }^{1,2}$, Mingyi $\mathrm{Ju}^{1,2}$, Baohui $\mathrm{Hu}^{1,2}$, Minjie Wei ${ }^{1,2}$, Miao $\mathrm{He}^{1,2 *}$ and Lin Zhao ${ }^{1,2 *}$

${ }^{1}$ Department of Pharmacology, School of Pharmacy, China Medical University, Shenyang, China, ${ }^{2}$ Liaoning Key Laboratory of Molecular Targeted Anti-tumor Drug Development and Evaluation, Liaoning Cancer Immune Peptide Drug Engineering Technology Research Center, Key Laboratory of Precision Diagnosis and Treatment of Gastrointestinal Tumors, Ministry of Education, China Medical University, Shenyang, China
\end{abstract}

Esophageal cancer (ESCA) is a leading cause of cancer-related mortality, with poor prognosis worldwide. DNA damage repair is one of the hallmarks of cancer. Loss of genomic integrity owing to inactivation of DNA repair genes can increase the risk of cancer progression and lead to poor prognosis. We aimed to identify a novel gene signature related to DNA repair to predict the prognosis of ESCA patients. Based on gene expression profiles of ESCA patients from The Cancer Genome Atlas and gene set enrichment analysis, 102 genes related to DNA repair were identified as candidates. After stepwise Cox regression analysis, we established a five-gene prognostic model comprising DGCR8, POM121, TAF9, UPF3B, and BCAP31. Kaplan-Meier survival analysis confirmed a strong correlation between the prognostic model and survival. Moreover, we verified the clinical value of the prognostic signature under the influence of different clinical parameters. We found that small-molecule drugs (trametinib, selumetinib, and refametinib) could help to improve patient survival. In summary, our study provides a novel and promising prognostic signature based on DNA-repair-related genes to predict survival of patients with ESCA. Systematic data mining provides a theoretical basis for further exploring the molecular pathogenesis of ESCA and identifying therapeutic targets.

Keywords: prognostic biomarkers, DNA repair, overall survival, esophageal cancer, small molecular drugs, targeted therapy

\section{INTRODUCTION}

Esophageal cancer (ESCA) is the sixth leading cause of cancer-related deaths worldwide, and its mortality has continued to increase [1]. ESCA has a poor prognosis due to early metastasis, and a 5years overall survival (OS) rate is around $15 \%[2,3]$. Even in the same cancer stage of ESCA patients, patient prognosis may be different. Therefore, it is imperative to construct prognostic biomarkers that can be used to judge the survival outcomes of patients with ESCA. Clinical oncologists can also use these markers to determine whether adjuvant treatment is needed. Owing to various genetic and phenotypic alterations that have been reported in ESCA, gene biomarkers have gradually become a cost-effective and precise method for predicting the prognosis of ESCA patients [4]. However, 
polymorphisms of genes and tumor heterogeneity mean that single-gene biomarkers are inadequate [5]. Thus, the search for prognostic markers in cancer patients has increasingly focused on multi-gene biomarkers [6].

Gene expression analysis can provide a means of identifying potential prognostic markers related to survival. In recent years, many studies have shown that various gene changes precede deterioration in prognosis in ESCA patients. Importantly, it has been reported that genomic DNA is highly susceptible to damage and can be influenced by different types of chemotherapy drugs. The genomic instability induced by DNA damage can result in cell apoptosis and tumorigenesis. The DNA repair process is often blocked or destroyed in cancer cells, enabling them to rapidly evolve and adapt, which ultimately drives the development of cancer lesions and metastasis [7]. In addition, defective DNA repair genes can promote cell aging, apoptosis and proliferation, make carriers prone to cancer [8], and change the sensitivity of cancers to chemotherapy. Therefore, DNA damage repair, as one of the hallmarks of cancer, is indispensable for maintaining the genomic integrity of the cell. Recent studies have identified single biomarkers related to DNA repair in ESCA or its subtypes that could predict patients' prognosis [9-11]. However, there is limited evidence regarding combined biomarkers of genes related to DNA repair in ESCA. Therefore, there is an urgent need to construct a prognostic gene signature based on DNA repair pathways for use in patients with ESCA.

The Cancer Genome Atlas (TCGA) is an authoritative, largescale collaborative work led by the National Cancer Institute and the National Human Genome Institute [12]. It can be used to analyze genomic and epigenetic changes in 33 human cancers at the DNA, RNA, protein, and epigenetic levels, thus supporting new discoveries and accelerating research progress to improve cancer diagnosis, treatment, and prevention [13]. TCGA provides a valuable resource for the cancer research community. It collects a large number of human cancer samples and normal tissues, enabling researchers to identify important genomic changes that may have key roles in the development of cancer, and facilitates deeper and broader research of the cancer genome [14]. Here, we analyzed ESCA data in TCGA to find reliable prognostic markers, and randomly divided the entire TCGA dataset into two groups for supplementary verification.

Based on TCGA data mining, we selected five genes (DGCR8, POM121, TAF9, UPF3B, and BCAP31) associated with DNA repair to construct a prognostic signature, and showed that this signature performed well in predicting the prognosis of patients. The results of the high-throughput data mining showed that our prognostic model could independently predict ESCA patients' survival. The results also provide a theoretical basis for further exploring the molecular pathogenesis of ESCA and identifying therapeutic targets.

\section{MATERIALS AND METHODS}

\section{Data Acquisition and Pre-Processing}

TCGA (https://cancergenome.nih.gov/, data release v23.0), a publicly available database, can be used for genomic analyses of 33 cancers (tumor samples and normal samples). We downloaded RNA expression data (fragments per kilobase million, FPKM) of 171 samples from the TCGA data portal. FPKM is a normalized estimation based on RNA sequencing data. The final expression levels of the FPKM data were determined by quantile normalization and $\log _{2}$ transformation using the "limma" R package. We also downloaded clinical information for all samples. We removed one sample owing to incomplete clinical information, leaving 170 samples (159 tumor samples and 11 normal samples) for further analysis. The clinical information included patients' general characteristics (age, gender, and race), subtype of ESCA, survival status, pathologic stage (TNM), neoplasm status, tumor location, neoplasm histological grade, residual tumor status and others (Table 1). We also downloaded an independent dataset (accession number GSE38129; $n=60,30$ normal and 30 tumor) from the Gene Expression Omnibus database (https://www.ncbi.nlm.nih.gov/geo/) for external validation. The platform of this dataset was GPL571. These data were normalized by robust multi-array average to validate the results.

\section{Screening DNA-Repair-Related Genes by Gene Set Enrichment Analysis}

The gene set enrichment analysis (GSEA, http://www. broadinstitute.org/gsea/index.jsp) included 1320 gene sets and showed its distinction in gene detection by testing gene sets but not individual gene. It was determined whether a given gene pathway shows statistically significant differences between a cancer group and a normal group [15, 16]. Here, we used GSEA to identify significant differences in DNA repair pathways between the ESCA group and the normal group, using gene expression profile data for ESCA. We also obtained 102 DNA-repair-related genes as candidates for further analysis.

For deeper analysis, we constructed a protein-protein interaction network for these 102 genes using Metascape (http://metascape.org) [17], which provides biological pathways obtained through independent and orthogonal experiments on datasets of more than 40 knowledgebase. $p<$ 0.05 is generally considered to represent significantly enriched pathways. Using molecular complex detection (MCODE), it can identify closely related protein groups, with biological function annotations for each group. We then explored the relationships between the 102 DNA-repair-related genes and biological pathways using gene ontology (GO) and Kyoto Encyclopedia of Genes and Genomes (KEGG) enrichment analysis with Metascape.

\section{Identification of DNA-Repair-Related Genes and Construction of Prognostic Model}

In order to identify survival-related genes in DNA repair gene sets, univariate Cox linear proportional hazard regression (PHR) analysis was performed with the "univariate" R package. Furthermore, in order to identify independent prognostic factors and construct a prognostic model, we performed multivariate Cox linear PHR analysis with the 
TABLE 1 | Summary of clinical characteristics of ESCA patients in three cohorts.

Characteristic

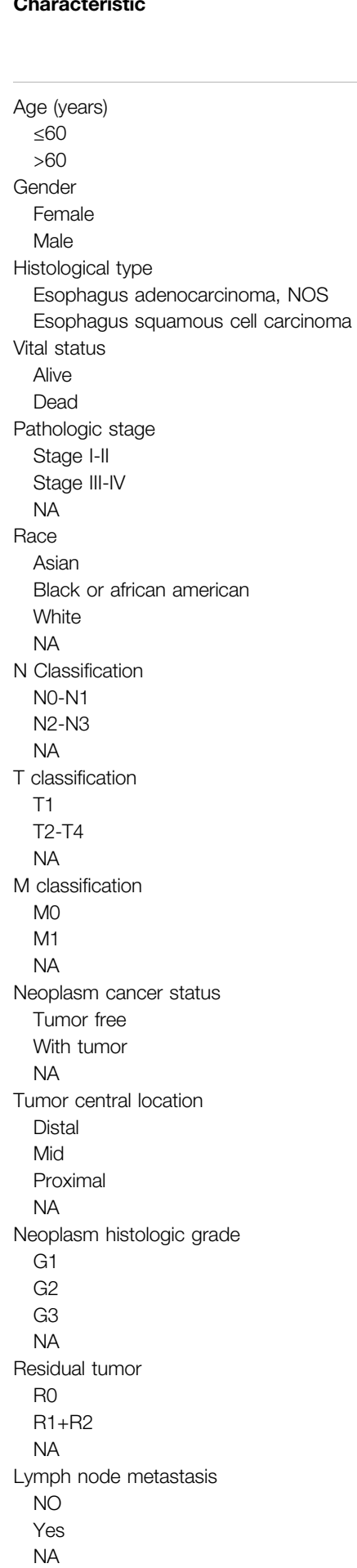

\section{Patients in entire \\ TCGA set \\ (n = 159), $n(\%)$}

Patients in subgroup

1 ( $n=79$ ),

n (\%)

81 (50.94\%)

78 (49.06\%)

$23(14.47 \%)$

136 (85.53\%)

79 (49.69\%)

$80(50.31 \%)$

$96(60.38 \%)$

63 (39.62\%)

87 (54.72\%)

68 (42.77\%)

$4(2.51 \%)$

38 (23.90\%)

$5(3.14 \%)$

$98(61.64 \%)$

$18(11.32 \%)$

133 (83.65\%)

$14(8.80 \%)$

$12(7.55 \%)$

25 (15.72\%)

132 (83.02\%)

$2(1.26 \%)$

$126(79.25 \%)$

$15(9.43 \%)$

18 (11.32\%)

$91(57.23 \%)$

58 (36.48\%)

$10(6.29 \%)$

111 (69.81\%)

$41(25.79 \%)$

$6(3.77 \%)$

$1(0.63 \%)$

$16(10.06 \%)$

65 (40.88\%)

43 (27.05\%)

35 (22.01\%)

119 (74.84\%)

$13(8.18 \%)$

27 (16.98\%)

83 (52.20\%)

$43(27.04 \%)$

33 (20.76\%)
44 (55.70\%)

35 (44.30\%)

$13(16.46 \%)$

66 (83.54\%)

36 (45.57\%)

43 (54.43\%)

47 (59.49\%)

32 (40.51\%)

46 (58.23\%)

32 (40.51\%)

$1(1.26 \%)$

22 (27.85\%)

$2(2.53 \%)$

$50(63.29 \%)$

5 (6.33\%)

65 (82.28\%)

7 (8.86\%)

$7(8.86 \%)$

$14(17.72 \%)$

$63(79.75 \%)$

2 (2.53\%)

$59(74.68 \%)$

$7(8.86 \%)$

$13(16.46 \%)$

49 (62.03\%)

26 (32.91\%)

$4(5.06 \%)$

54 (68.35\%)

$22(27.85 \%)$

$3(3.80 \%)$

$0(0.00 \%)$

$6(7.6 \%)$

$33(41.77 \%)$

$23(29.11 \%)$

17 (21.52\%)

60 (75.95\%)

$3(3.80 \%)$

16 (20.25\%)

34 (43.04\%)

27 (34.18\%)

$18(22.78 \%)$
Patients in subgroup

$2(n=80)$,

n (\%)

37 (46.25\%)

43 (53.75\%)

$10(12.50 \%)$

70 (87.5\%)

43 (53.75\%)

37 (46.25\%)

49 (61.25\%)

31 (38.75\%)

$41(51.25 \%)$

36 (45.00\%)

3 (3.75\%)

$16(20.00 \%)$

3 (3.75\%)

48 (60.00\%)

13 (16.25\%)

68 (85.00\%)

7 (8.75\%)

5 (6.25\%)

$11(13.75 \%)$

69 (86.25\%)

$0(0.00 \%)$

67 (83.75\%)

$8(10.00 \%)$

$5(6.25 \%)$

42 (52.50\%)

32 (40.00\%)

$6(7.5 \%)$

57 (71.25\%)

$19(23.75 \%)$

3 (3.75\%)

$1(1.25 \%)$

$10(12.5 \%)$

$32(40.00 \%)$

$20(25.00 \%)$

$18(22.50 \%)$

$59(73.75 \%)$

$10(12.50 \%)$

$11(13.75 \%)$

$49(61.25 \%)$

$16(20.00 \%)$

$15(18.75 \%)$

Abbreviations: ESCA, esophageal cancer; NA, not available.

"multivariate" R package. Finally, we constructed a prognostic signature comprising five genes that could predict the prognosis of ESCA patients. Based on gene expression values and regression coefficients, we developed a risk scoring system to predict the survival of patients. The equation is as follows 


$$
\text { Risk score }=\sum_{i}^{n} \operatorname{Exp} i * \beta i
$$

where Exp represents the gene expression level, and $\beta$ is the partial regression coefficient of independent variables for each gene. We ranked the patients into two groups (high and low risk) using the median risk value.

Furthermore, we performed deeper analysis of the five genes using GeneMANIA (http://www.genemania.org), which can identify functionally similar genes using a wealth of genomics and proteomics data and indicate the function of these genes [18]. We uploaded the selected genes to GeneMANIA to identify interacting genes and analyze gene functions. Mutational analysis was carried out, and the drug sensitivities and biological functions of the five genes were examined using GSCALite (http://bioinfo.life.hust.edu.cn/ web/GSCALite/) [19], which is widely used for gene set analysis in various cancers. The structures of potential drug molecules were visualized using PubChem (https://pubchem. ncbi.nlm.nih.gov/). Alterations of the five genes in ESCA were shown with cBioPortal (http://www.cbioportal.org/).

\section{Validation of Five-Gene Prognostic Signature in ESCA Patients}

The entire dataset of TCGA patients with ESCA $(n=159)$ were randomly separated into two subgroups, denoted TCGA subgroup 1 ( $n=79$; Table 1$)$ and TCGA subgroup $2(n=80$; Table 1). The prognostic signature was identified in the entire TCGA dataset and validated in all three groups (the TCGA entire group and the two subgroups). Using the risk score formula, we calculated the risk value for each patient, and divided patients into two (high and low) groups by the median value. In order to validate the predictive capability of the prognostic signature, Kaplan-Meier (K-M) survival analysis (using the "survival" R package) was performed to compare differences in OS. Time-dependent receiver operating characteristic (ROC) curves were also constructed to evaluate the prognostic accuracy of the model. Likewise, we used stepwise Cox linear regression analysis to investigate the influence of clinical parameters on the prognostic signature with the survival package in $\mathrm{R}$. Next, we used stepwise Cox linear PHR analysis to select clinical factors with prognostic characteristics using $\mathrm{R}$ programs.

\section{Statistical Analysis}

For all data in our study, prognostic indicators to predict patient survival were filtered out using the corresponding $\mathrm{R}$ packages ( $\mathrm{R}$ version 3.5.2). K-M survival curves with twosided log-rank test were used to estimate the probability of survival. Differential expression of genes was plotted using GraphPad Prism (version 8.0). Statistical analysis was performed using IBM SPSS 25.0. An independent $t$-test was used to compare differences, with $p$ value $<0.05$ was represented significance.

\section{RESULTS}

\section{Selection of DNA-Repair-Related Genes in ESCA Patients}

The detailed workflow of this study is shown in Figure 1. To obtain DNA-repair-related genes, we uploaded 57,072 genes for TCGA-ESCA patients $(n=159)$ to GSEA. Next, we collected 102 genes with $p<0.001$ that made the greatest contributions to the DNA repair pathway (ESM1: Supplementary Table 1) according to GSEA. The enrichment plot showed that there were statistically significant differences in the identified gene set between the ESCA group and the normal group (Figure 2A). In addition, we analyzed the protein interactions of these genes (Figure 2B, ESM1: Supplementary Table 2). According to the MCODE algorithm, there are three main modes that provide potential value for protein analysis. Biological process enrichment analyses for GO categories and KEGG pathways (Figure 2C) were carried out using the Metascape website. We found that these 102 genes were related to aspects of the DNA repair pathway, including nucleotide-excision repair, DNA-template transcription and termination, damaged DNA binding, base excision repair, nucleotide biosynthetic process, nucleoside metabolic process, and mitotic cell cycle phase transition.

Furthermore, we analyzed the correlation of gene expression with OS based on univariate Cox PHR analysis. As some genes may not have been independent indicators, we applied multivariable Cox PHR analysis to identify the most effective genes. Finally, a five-gene prognostic model comprising DGCR8, POM121, TAF9, UPF3B, and BCAP31 was screened as an independent prognostic biomarker for ESCA patients. We also obtained the hazard ratio (HR, instant probability of reaching alignment) of each gene, as shown in Table 2. For further analysis, we classified these five genes as risk type $(\mathrm{HR}>1)$ or protective type $(\mathrm{HR}<1)$. Therefore, BCAP31, TAF9, and UPF3B were risk-related genes, as their high expression was associated with shorter survival time, whereas DGCR8 and POM121 were protective genes whose high expression meant longer survival time.

In addition, we used GeneMANIA to predict interacting genes and their functions. The results showed that DGCR8 and Drosha (an rnase enzyme) had the strongest correlation (Figure 2D). Notably, both DGCR8 and Drosha have been shown to play important and irreplaceable parts in ultraviolet (UV)-induced DNA damage repair [20]. This also confirmed that the genes we had selected were suitable to construct a robust prognostic model. Besides, pie chart (Figure 2E) was performed to assess the possible mechanisms involving these genes. The results showed that all five genes were related to the cell cycle and DNA damage and could regulate the PI3K/AKT pathway, indicating that they have critical roles in cancer.

\section{Mutation and Differential Expression Analysis of Five Genes in Signature}

First, we analyzed the alterations of the five genes in different cancers using Metascape. We found that mutations of these genes 


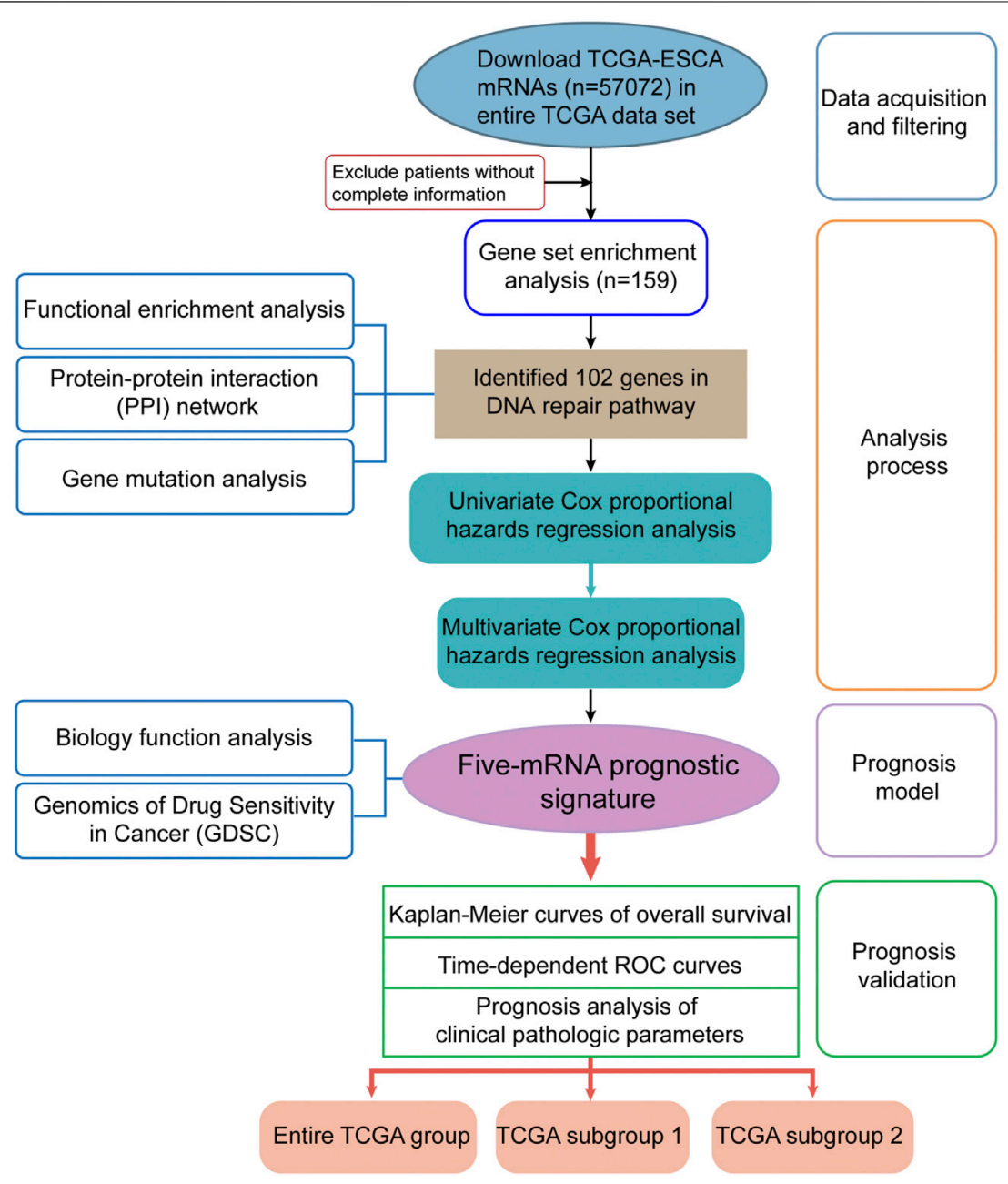

FIGURE 1 | Flow diagram of data and analyses in this work.

occurred in various cancers, including ESCA (Figure 3A). Then, we analyzed the changes in the five genes in ESCA samples using the cBioPortal database. For the protective-type genes (DGCR8 and POM121), 11 and $15 \%$ of patients showed alterations. For the risk-type genes (UPF3B, BCAP31, and TAF9), 13, 11, and 14\% of patients, respectively, showed changes (Figure 3B). These results suggest gene changes may be one research object.

Subsequently, we evaluated the gene alterations in two subtypes including esophageal squamous cell carcinoma (ESCC) and esophageal adenocarcinoma (EAD). Gene alterations in these two subtypes included mutation, amplification, deep deletion, up-regulation, down-regulation and multiple alterations (Figure 3C). The results suggest no significant difference between ESCC and EAD in this regard.

We also compared the expression of the selected five genes in the tumor group $(n=159)$ and the normal group $(n=11)$, and showed that they were significantly up-regulated in tumor tissues $(p<0.05$, Figure 3D). In addition, in order to further verify that there was significant differential expression of the five genes in the prognostic signature between normal and tumor samples, we performed validation in a independent dataset-GSE38129. As shown in ESM2: Supplementary Figure 1, all five genes were differentially expressed in GSE38129, and the differences were statistically significant $(p<0.05)$.

\section{Construction of Five-Gene Prognostic Signature}

Based on the results of the multivariable Cox PHR analyses, the five genes were used to establish a risk scoring system. We used the risk score formula to calculate a risk score for each patient, and ranked the patients into low- and high-risk groups in the three cohorts according to the median risk score value (Figures $\mathbf{4 A - C}$ ). We also constructed scatter plots of patient survival time to visualize the survival status of ESCA patients in the three cohorts (Figures 4D-F). Comparison of the two (low and high) risk groups showed that patients with higher risk scores had higher mortality and lower survival rates. In addition, a heatmap (Figures 4G-I) was used to illustrate the expression profile of the five-gene signature. Overall, the results indicate that the risk score had good potential to predict patients' prognosis. 


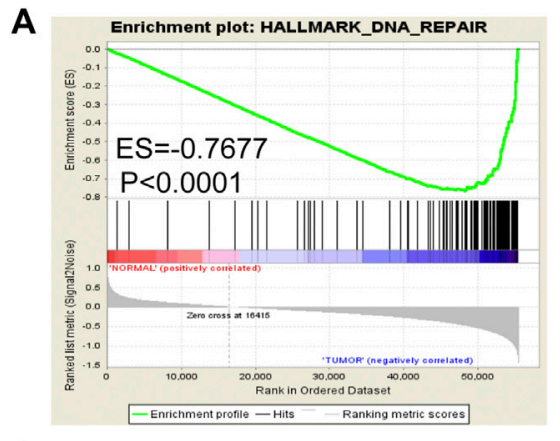

C

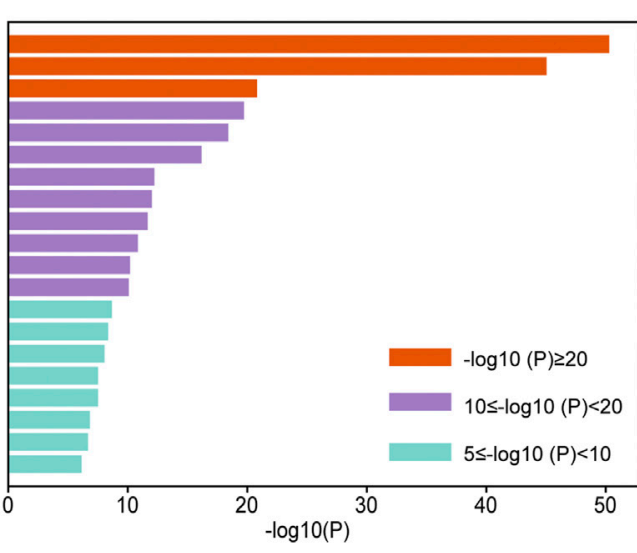

B
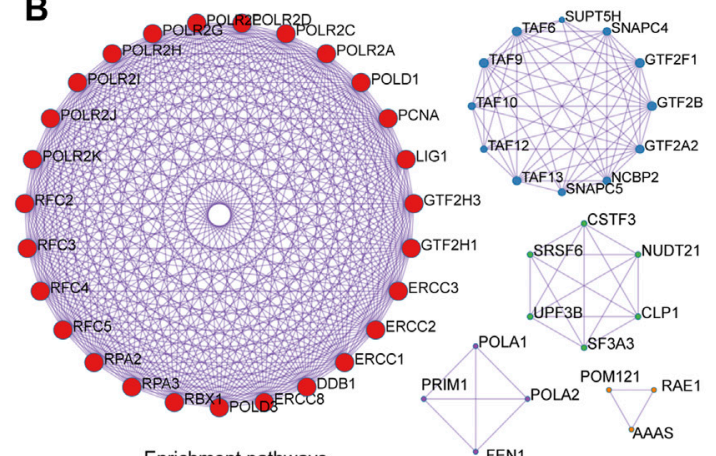

Enrichment pathways

Nucleotide-excision repair

Nuclear DNA-directed RNA polymerase complex

DNA-templated transcription, termination

Transcription factor TFIID complex

Nucleotide-excision repair, preincision

complex stabilization

Damaged DNA binding

Deoxyribonucleoside triphosphate metabolic process

Base excision repair

DNA strand elongation involved in DNA replication

Nucleobase-containing small molecule biosynthetic process

Single-stranded DNA binding

Nucleotide biosynthetic proces

Nucleoside metabolic process

Promoter-specific chromatin binding

mRNA 3'-end processing

Mitotic cell cycle phase transition

Nucleobase-containing compound kinase activity

intracellular transport of virus

UV protection

Cul4B-RING E3 ubiquitin ligase complex

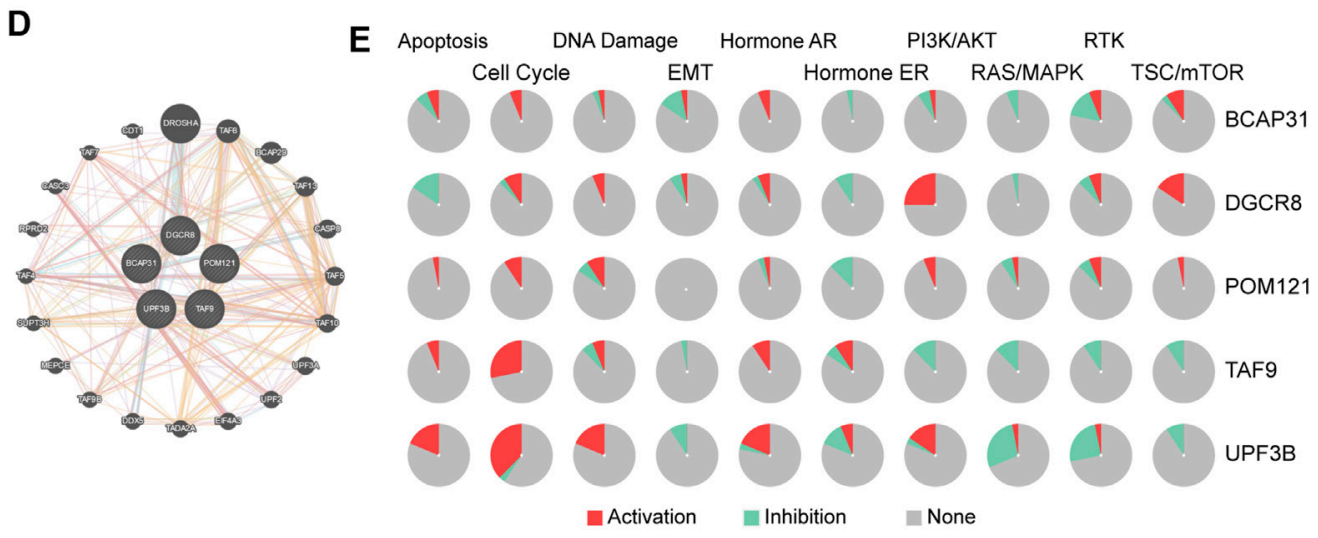

FIGURE 2 | Screening of genes related to DNA repair in ESCA. (A) Enrichment plots showing differential expression of DNA-repair-related genes in normal tissues $(n=11)$ and tumor tissues $(n=159)$ according to GSEA. (B) Protein-protein interaction network $(n=102)$. (C) Functional enrichment (GO and KEGG) analyses of DNA repair genes ( $n=102)$. (D) Interaction of five genes by GeneMANIA. (E) Biological function analysis of the individual five genes in ESCA. Abbreviation: ESCA, esophageal carcinoma; GSEA, gene set enrichment analysis; GO, Gene Ontology; KEGG, Kyoto Encyclopedia of Genes and Genomes.

TABLE 2 | The detailed information of selected five genes related to overall survival in patients with ESCA.

\begin{tabular}{lcccc}
\hline Gene & Ensemble ID & B (cox) & HR & p-value \\
\hline BCAP31 & ENSG00000185825.14 & 0.440 & 1.938 & 0.0046 \\
TAF9 & ENSG00000273841.3 & 0.397 & 1.683 & 0.0243 \\
UPF3B & ENSG00000125351.9 & 0.383 & 1.657 & 0.0048 \\
POM121 & ENSG00000196313.10 & -0.373 & 0.603 & 0.0338 \\
DGCR8 & ENSG00000128191.12 & -0.864 & 0.408 & 0.0070 \\
\hline
\end{tabular}

Abbreviations: ESCA, esophageal cancer; HR, hazard ratio.
Next, we analyzed the clinicopathological parameters by stepwise Cox PHR analysis to determine whether the five-gene risk model functioned as an independent prognostic signature when adjusted for cancer stage, stage- $\mathrm{M}$ and residual tumor (Table 3). As shown in results, univariate Cox PHR analysis pointed out that five-gene prognostic signature and these clinical pathological factors indeed have prognostic value at the aspect of predicting survival of patients with ESCA. Importantly, five-gene signature, cancer stage and stage $\mathrm{M}$ were also independent prognostic indicators with significant 


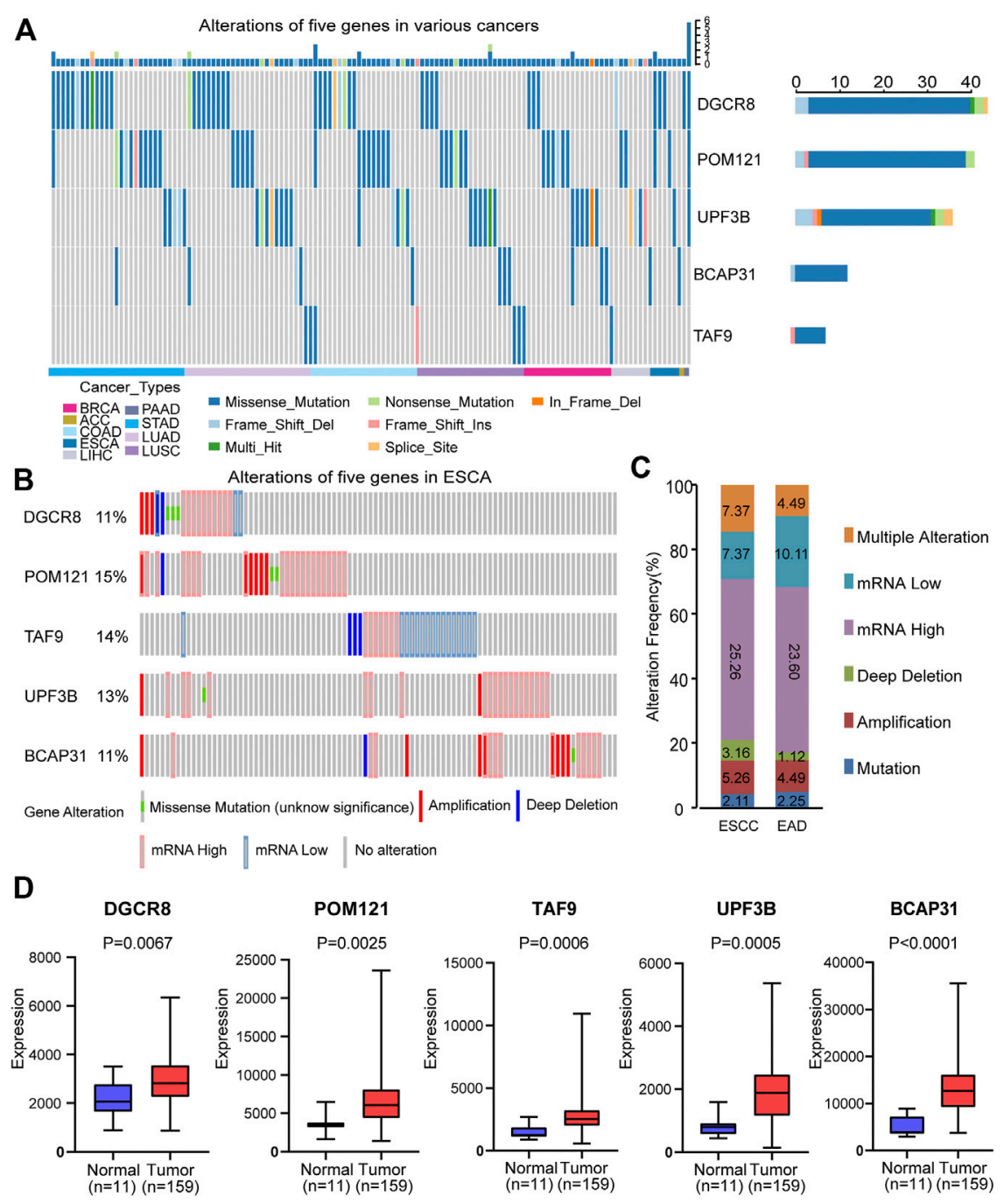

FIGURE 3 | Alterations and differential expression of the five genes. (A) Alterations of the five genes in different cancers. (B) Alterations of the five genes in ESCA patients. (C) Genomic alterations of the five genes in patients with ESCA subtypes. (D) Differential expression of the selected five genes in normal group ( $n=11$ ) and tumor group $(n=159)$. Two-sided log-rank and Wilcoxon $p<0.05$ were considered significant. Abbreviation: ESCA, esophageal carcinoma; ESCC, esophageal squamous carcinoma; EAD, esophageal adenocarcinoma.

differences $(p<0.05)$ both in univariate and multivariate Cox analysis. In particular, risk score had the strongest predictive ability among these indicators (HR 3.388; 95\% confidence interval (CI) 1.664-6.899, $p=0.001)$. These results demonstrate that the five-gene signature can effectively predict the prognosis of patients with ESCA and prognostic independent of other clinical factors.

\section{Validation of the Prognostic Efficiency of the Five-Gene Signature in Three Cohorts}

We randomly divided all the TCGA-ESCA tumor samples into two subgroups. As well as validation in the entire TCGA group, we validated the prognostic signature using survival curves in these two subgroups. K-M survival curves plotted in the entire TCGA dataset $(n=159)$ showed that the prognostic model stratified patients by OS with significant differences, and the survival rate of high-risk patients was lower than that of low-risk patients $(p<0.0001$; Figure 5A). The area under the curve (AUC) of the ROC curves showed that the five-gene signature had good predictive performance for ESCA patients $(\mathrm{AUC}=0.759$; Figure 5B). In the TCGA subgroup $1(n=79)$, the K-M survival curve ( $p=0.0021$, Figure 5C) and ROC curve (AUC $=0.733$; Figure 5D) also demonstrated that the five-gene model was able to predict the prognosis of ESCA patients. In TCGA subgroup $2(n=80)$, the K-M survival curve $(p=0.0017$, Figure 5E) and ROC curve (AUC $=0.711$; Figure 5F) again validated the model. Compared with any of the individual genes (ESM2: Supplementary Figure 2), the five-gene model had better predictive performance as a prognostic indicator in the entire TCGA dataset, with the lowest $p$ value $(p<0.0001)$. 


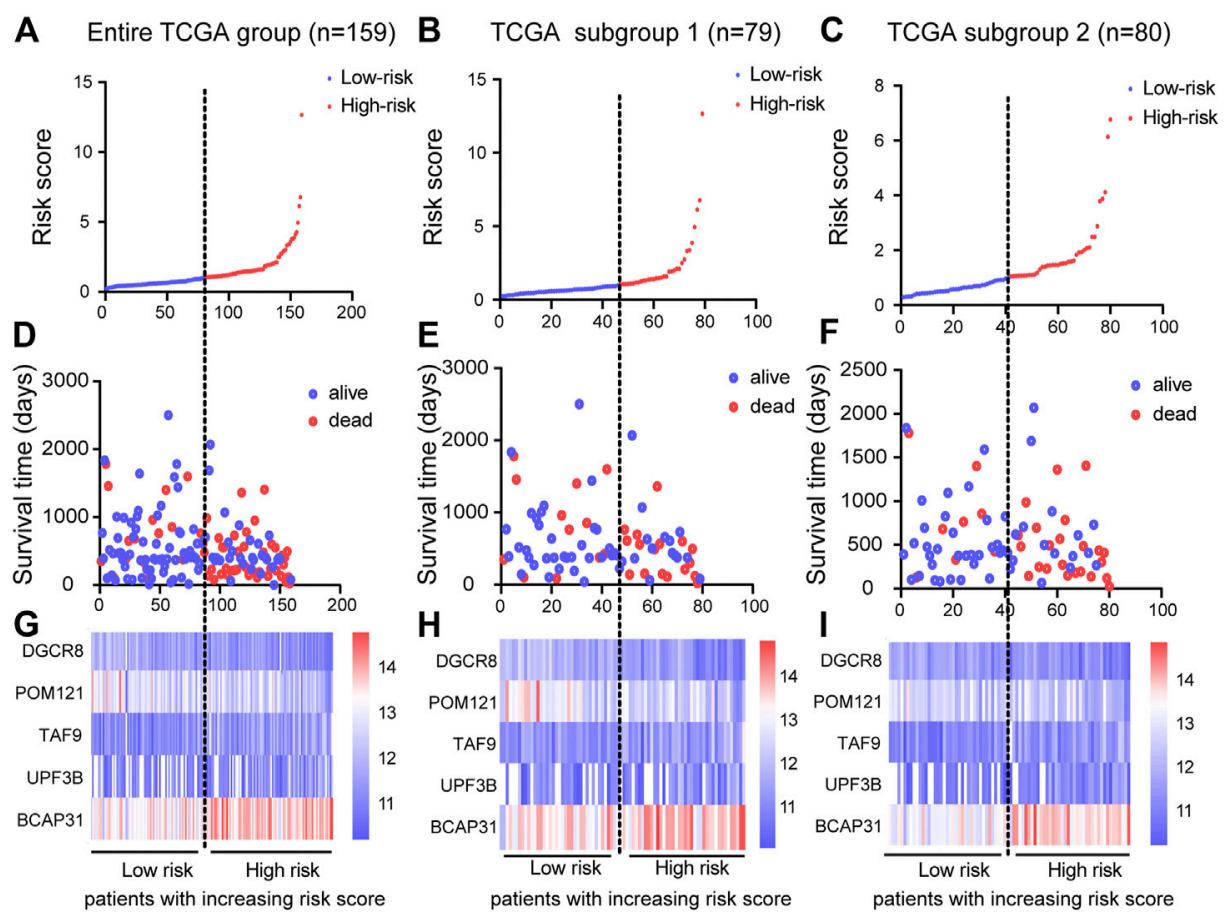

FIGURE 4 | Construction of prognostic risk score system and identification of five-gene prognostic model. Risk score distribution of five genes in three cohorts: entire TCGA group ( $n=159)$, TCGA subgroup $1(n=79)$, and TCGA subgroup $2(n=80)$. Top (A)-(C) and middle (D)-(F) plots show patient survival time and status based on risk score system (G)-(I) Heatmap of expression of the five genes; color from blue to red illustrates a trend from low expression to high expression.

TABLE 3 | Univariable and multivariable Cox linear regression analysis for risk score and different clinical pathological parameters.

\begin{tabular}{|c|c|c|c|c|c|c|c|}
\hline \multirow[b]{2}{*}{ Clinical feature } & \multirow[b]{2}{*}{ Number } & \multicolumn{3}{|c|}{ Univariable analysis } & \multicolumn{3}{|c|}{ Multivariable analysis } \\
\hline & & HR & $95 \% \mathrm{Cl}$ of $\mathrm{HR}$ & $p$ value & HR & $95 \% \mathrm{Cl}$ of $\mathrm{HR}$ & $p$ value \\
\hline Risk score (low/high) & $79 / 80$ & 3.819 & $2.161-6.748$ & $<0.0001$ & 3.388 & $1.664-6.899$ & 0.001 \\
\hline Cancer stage (stage I-II/III-IV) & $87 / 68$ & 3.182 & $1.774-5.710$ & $<0.0001$ & 2.732 & $1.328-5.623$ & 0.006 \\
\hline Stage-M (M0/M1) & $126 / 15$ & 4.92 & $2.243-10.794$ & $<0.0001$ & 2.535 & $1.024-6.276$ & 0.044 \\
\hline Residual tumor (R0/R1+ R2) & $119 / 13$ & 2.324 & $1.143-4.724$ & 0.020 & 1.199 & $0.539-2.668$ & 0.657 \\
\hline
\end{tabular}

Abbreviations: HR, hazard ratio; Cl, confidence interval.

\section{Validation of Independent Prognostic Indicator Under the Influence of Clinical Pathological Factors in Entire TCGA Cohort}

We carried out further stratified analyses of clinical factors to investigate the clinical value of the prognostic model in the entire TCGA dataset. The results showed that the five-gene signature related to DNA repair was an independent prognostic indicator for patients with ESCA, compared with cancer stage (stage I-II or stage III-IV, Figure 6A), residual tumor status (R0 or R1+R2, Figure 6B), cancer status (tumor free or with tumor, Figure $6 \mathrm{C}$ ) and lymph node metastasis (no or yes, Figure 6D). But there were no reference values of $\mathrm{K}-\mathrm{M}$ curves for stage $M$ because of the uneven case numbers of patients. As shown in results, the five-gene signature, as well as having good prognostic value, could serve as an independent prognostic indicator in ESCA patients.
In order to explore molecules that could serve as targeted drugs, we analyzed the drug sensitivity of the five genes in the prognostic signature. As shown in Figure 7A, UPF3B and BCAP31 are more sensitive to drugs. Potential targeted drugs were identified, including trametinib, selumetinib, and refametinib, which could be used to improve patient survival. Based on Spearman correlation analysis, we determined the top three drugs (Figure 7B) with potential for further clinical research.

\section{DISCUSSION}

ESCA is one of the most aggressive cancers, with overall mortality as high as $88 \%$ [21]. Although advances in therapeutics have improved clinical outcomes to some extent, the survival rate remains poor. Many biomarkers have been found to be related to survival, and accumulating evidence indicates that gene biomarkers are the 


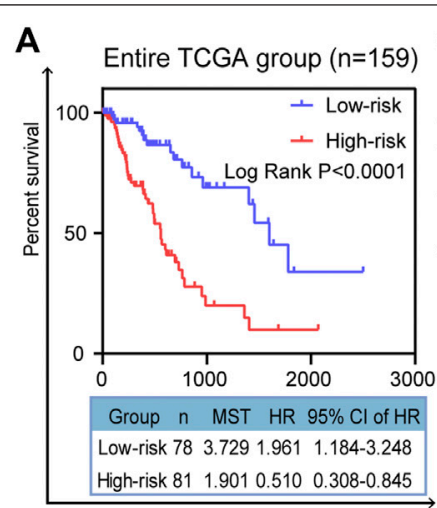

Overall survival (days)

B

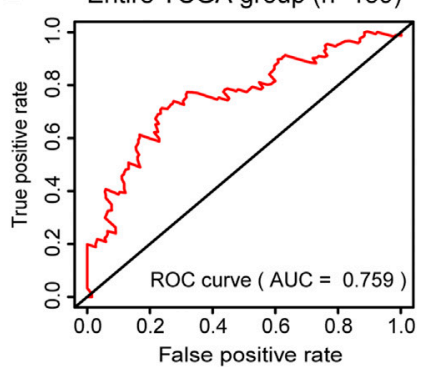

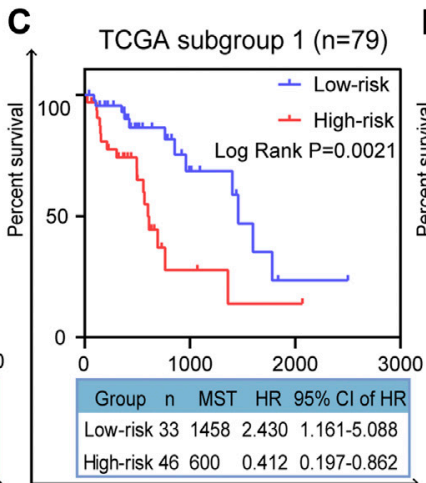

Overall survival (days)

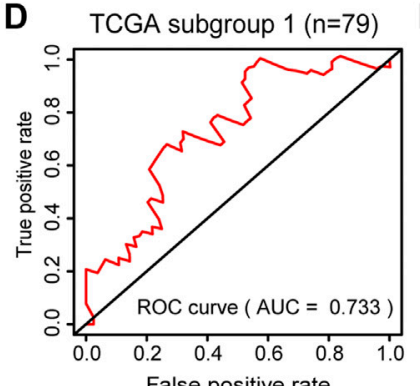

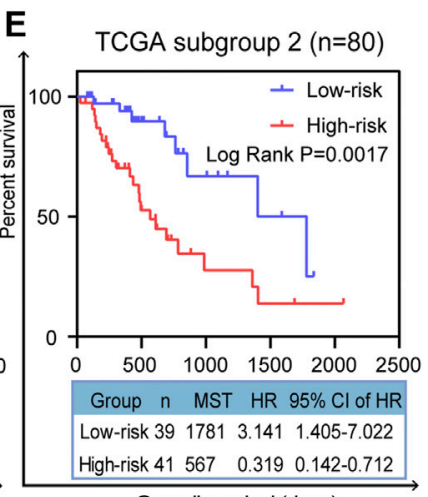

Overall survival (days)

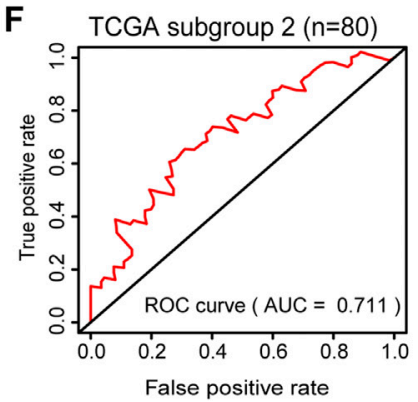

FIGURE 5 | Validation of prognostic signature for patients with ESCA. K-M survival curves for prognostic model and time-dependent ROC curve for (A, B) entire TCGA group ( $n=159)$, (C, D) TCGA subgroup $1(n=79)$, and $(\mathbf{E}, \mathbf{F})$ TCGA subgroup $2(n=80)$. Two-sided log-rank and Wilcoxon $p<0.05$ were considered significant. Abbreviation: MST, median survival time.

preferred way to predict prognosis. Therefore, there is an urgent need to investigate the gene expression profile of ESCA, in order to be able to better assess the prognosis of ESCA patients. Establishing and validating prognostic gene biomarkers may improve clinical outcomes for these patients in the near future.

Recent studies have identified various single genes as biomarkers to reveal the relationship of patients' survival and cancer progression. For example, DLEU2 [22], FAM60A [23] and CENPE [24] were demonstrated to be independent biomarkers of unfavorable OS in ESCA patients. However, compared with combined markers, single biomarkers are insufficient to independently predict patient prognosis, which can be affected by various factors. Therefore, the application of combined markers in cancer has been reported in succession. For example, a signature of seven long non-coding RNAs (lncRNAs) could indicate survival in ESCC [25]. Integrated analysis led to identification of a three-gene model as a potential biomarker for ESCC [26]. Men and colleagues constructed an 11gene signature based on the TCGA database that could predict the OS of patients with ovarian cancer [27]. In breast cancer, a fivelncRNA signature has been identified as a prognostic biomarker [28]. Moreover, a prognostic signature including nine genes was shown to have good performance in predicting OS of colorectal cancer patients [29]. Therefore, multi-gene prognostic signatures are necessary for determining cancer prognosis.

DNA damage readily occurs during the cell cycle; it can disturb the cell's steady state and lead to mutations, cell death, and cancer [30]. In about half of cases, doxorubicin, cisplatin [31] and other chemotherapy drugs will cause huge damage to the DNA of normal cells as well as that of tumor cells during treatment, leading to a limited curative effect and poor prognosis. Notably, DNA repair, DNA damage detection point, transcription reaction and apoptosis are four ways to resume DNA damage. Defects in any of these pathways can lead to genomic instability and cancer. Therefore, DNA damage repair pathways must be considered in future cancer research. Gene markers related to these pathways may play an important part in prediction of patient survival and formulation of cancer treatment strategies. The single genes CD59 [9], RAP80 [10] and SOX17 [11] have been reported to serve as DNA-repair-related biomarkers to predict patients' prognosis in ESCA or subtypes of this cancer. However, such single-gene signatures are insufficient to predict prognosis. Therefore, we aimed to discover a multi-gene signature related to DNA repair for predicting the survival of ESCA patients.

In this study, through a comprehensive analysis, we developed a DNA-repair-related gene marker to predict the prognosis of patients with ESCA. The vast datasets of TCGA provide an opportunity to systematically analyze mRNA expression profiles in cancer. Therefore, we downloaded mRNA expression profiles for the TCGA-ESCA dataset to find markers that could predict patients' prognosis. We applied GSEA to identify DNA-repair-related mRNAs, which were subjected to univariate and multivariate Cox PHR analysis. In this way, we obtained a five-gene signature (DGCR8, POM121, TAF9, UPF3B, and BCAP31) as a novel prognostic model. Afterward, according to the Cox coefficient and gene expression values for 

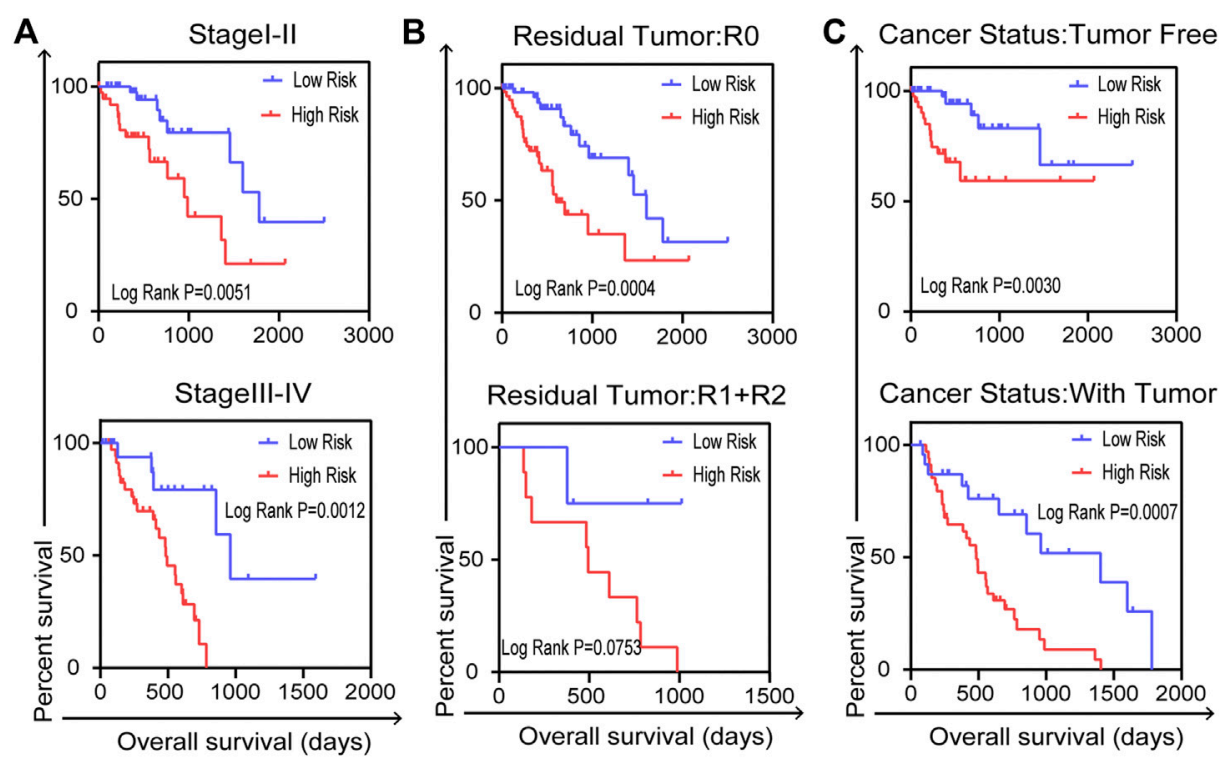

D Lymph Node Metastasis:NO
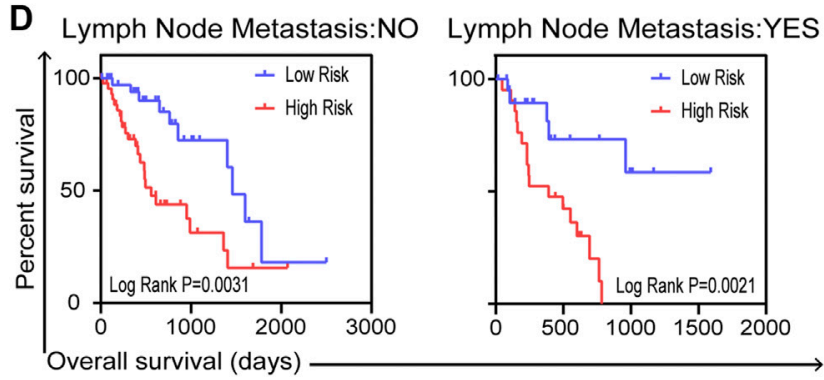

FIGURE 6 | Stratified analysis for further data mining. Validation of the five-gene prognostic signature in patients with ESCA for (A) cancer stage, (B) residual tumor, (C) cancer status and (D) lymph node metastasis in entire TCGA dataset $(n=159)$. Two-sided log-rank and Wilcoxon $p<0.05$ were considered significant.

each patient, a risk scoring system was established in the entire TCGA dataset. Then, we validated the prognostic model using K-M survival curves. The results showed that high-risk patients had a poorer survival rate compared with low-risk patients in the entire TCGA group and in the two subgroups. The AUC of the ROC curve for the five-gene signature was greater than 0.7 in these three cohorts, indicating the strong prognostic value of the signature. Subsequently, validation using clinical factors further indicated that the five-gene signature is an independent indicator in ESCA.

Notably, among the five genes, DGCR8 has been reported to have a critical role in DNA damage response and DNA repair. Studies have shown that DGCR8 together with Drosha (an rnase enzyme) can mediate the repair of UV-induced DNA lesions. Moreover, Swahari and colleagues found that deletion of DGCR8 resulted in DNA damage in the developing mouse brain [32]. DGCR8 is also associated with susceptibility to various cancers [33], including prostate cancer, Wilms tumor, and ovarian cancer. POM121 has been reported to be a key contributor to prostate cancer aggressiveness [34]. In addition, Guo et al [35] found that HIV-1 replication was significantly decreased by small interfering RNAmediated POM121 knockdown. TAF9 (TATA-binding protein) is one of several histone folding TAFs that maintain the structural integrity [36]. The p53 tumor suppressor gene modulates the activity of the GLI1 oncogene through interactions with the shared activator TAF9 [37]. UPF3B is part of a multi-protein complex that is involved in mRNA nuclear export and the initiation of nonsense-mediated mRNA decay (NMD). About $11 \%$ of human genetic diseases are due to $\mathrm{NMD}$, which produces premature translation termination codons in mRNAs. UPF3B has been identified as a potential treatment for NMD-induced diseases, including cancers [38]. BCAP31 (a member of the Bcl-2 protein family) has a potential function in cancer apoptosis, with a role in the proliferation and apoptosis of keratinocytes in cancers. BCAP31 has been reported to be upregulated in hepatocellular carcinoma [39]; similarly, in our study, BCAP31 was up-regulated in ESCA patients. Another study found that BCAP31 was related to patient survival in breast cancer [40]. However, the role of genes in ESCA patients should be further evaluated.

The advantages of our prognostic predictor are obvious. First, by multistep Cox PHR analysis, we identified a five-gene signature related to DNA repair and the risk coefficient of each patient, so as to build a risk score equation for ESCA patients to be recruited. Next, patients were assigned into two groups by the median risk value according to the equation. Based on the validation results for the clinical 
A Genomics of Drug Sensitivity in Cancer (GDSC)

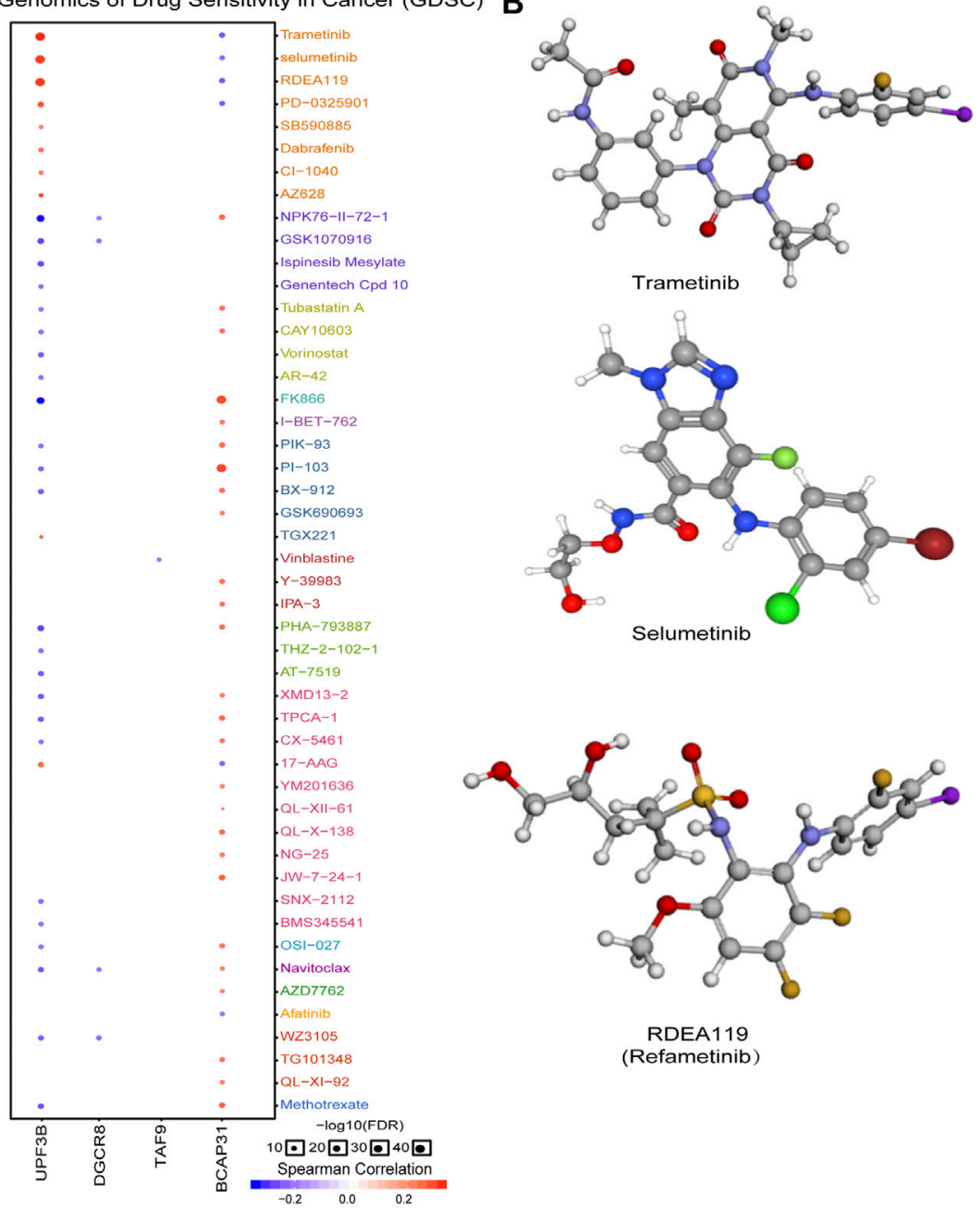

FIGURE 7|Analysis of potential drug sensitivity of five genes. (A) Genomics of drug sensitivity in cancer (GDSC). (B) Structure of potential targeted drugs including trametinib, selumetinib and refametinib.

pathological parameters, we confirmed that the five-gene signature could effectively predict the prognosis of patients under the influence of different clinical characteristics. This suggests it could predict patients' prognosis without considering other pathological parameters. The drug sensitivity analysis indicated that smallmolecule drugs have potential clinical value for improving patients' survival outcomes. Although further investigation and experimentation are needed to elucidate the biological mechanisms of the five-gene signature in ESCA development and progression, the prognostic value of the gene signature is promising.

\section{CONCLUSION}

In conclusion, we identified a novel five-gene predictive model comprising DGCR8, POM121, TAF9, UPF3B, and BCAP31 to indicate prognosis of patients based on integrated bioinformatics analysis. Our study explored the potential clinical significance of this biomarker. The results of the high-throughput data mining show that our prognostic model could independently predict ESCA patients' survival. These results also provide a theoretical basis for further exploring the molecular pathogenesis of ESCA and identifying therapeutic targets.

\section{DATA AVAILABILITY STATEMENT}

The original contributions presented in the study are included in the article/Supplementary Material, further inquiries can be directed to the corresponding authors.

\section{AUTHOR CONTRIBUTIONS}

Writing-original draft preparation: LW and XL. Methodology: LZ, LJ and XS. Formal analysis and investigation: $\mathrm{AQ}, \mathrm{TC}, \mathrm{MJ}$ and $\mathrm{BH}$; 
Writing-review and editing: $\mathrm{MW}, \mathrm{MH}$ and LZ. All authors critically reviewed the manuscript in its entirety and approved the final content.

\section{FUNDING}

This work was supported by Grants from Liaoning Revitalization Talents Program (No. XLYC1807201), Major Spcial S\&T Projects in Liaoning Province (2019JH1/10300005), National Natural Science Foundation of China (No. 81903658, 81703560), Liaoning Province Scientific Research Foundation (No. JC2019032) and Shenyang S\&T Projects (No. 19-109-4-09, 20-204-4-22).

\section{REFERENCES}

1. Ferlay J, Soerjomataram I, Dikshit R, Eser S, Mathers C, Rebelo M, et al. Cancer incidence and mortality worldwide: sources, methods and major patterns in GLOBOCAN 2012. Int J Cancer (2015). 136(5):E359-E386. doi:10.1002/ijc. 29210

2. Domper AM, Ferrandez AA, Lanas AA. Esophageal cancer: risk factors, screening and endoscopic treatment in Western and Eastern countries. World J Gastroenterol (2015). 21(26):7933-7943. doi:10.3748/wjg.v21.i26.7933

3. Lagergren J, Smyth E, Cunningham D, Lagergren P. Oesophageal cancer. The Lancet (2017). 390(10110):2383-2396. doi:10.1016/S0140-6736(17)31462-9

4. Januszewicz W, Fitzgerald RC. Early detection and therapeutics. Mol Oncol (2019). 13(3):599-613. doi:10.1002/1878-0261.12458

5. Nishizuka SS, Mills GB. New era of integrated cancer biomarker discovery using reverse-phase protein arrays. Drug Metab Pharmacokinet (2016). 31(1):35-45. doi:10.1016/j.dmpk.2015.11.009

6. Haisley KR, Hart CM, Kaempf AJ, Dash NR, Dolan JP, Hunter JG. Specific tumor characteristics predict upstaging in early-stage esophageal cancer. Ann Surg Oncol (2019). 26(2):514-522. doi:10.1245/s10434-018-6804-z

7. Pearl LH, Schierz AC, Ward SE, Al-Lazikani B, Pearl FMG. Therapeutic opportunities within the DNA damage response. Nat Rev Cancer (2015). 15(3):166-180. doi:10.1038/nrc3891

8. Ding L, Getz G, Wheeler DA, Mardis ER, McLellan MD, Cibulskis K, et al. Somatic mutations affect key pathways in lung adenocarcinoma. Nature (2008). 455(7216):1069-1075. doi:10.1038/nature07423

9. Zhou Y, Chu L, Wang Q, Dai W, Zhang X, Chen J, et al. CD59 is a potential biomarker of esophageal squamous cell carcinoma radioresistance by affecting DNA repair. Cell Death Dis (2018). 9(9):887. doi:10.1038/s41419-018-0895-0

10. Yang Q, Lin W, Liu Z, Zhu J, Huang N, Cui Z, et al. RAP80 is an independent prognosis biomarker for the outcome of patients with esophageal squamous cell carcinoma. Cel Death Dis (2018). 9(2):146. doi:10.1038/s41419-017-0177-2

11. Kuo I-Y, Huang Y-L, Lin C-Y, Lin C-H, Chang W-L, Lai W-W, et al. SOX17 overexpression sensitizes chemoradiation response in esophageal cancer by transcriptional down-regulation of DNA repair and damage response genes. J Biomed Sci (2019). 26(1):20. doi:10.1186/s12929-019-0510-4

12. Deng M, Brägelmann J, Schultze JL, Perner S. Web-TCGA: an online platform for integrated analysis of molecular cancer data sets. BMC Bioinformatics (2016). 17(1):72. doi:10.1186/s12859-016-0917-9

13. Weinstein JN, Collisson EA, Collisson EA, Mills GB, Shaw KRM, Ozenberger $\mathrm{BA}$, et al. The cancer genome Atlas pan-cancer analysis project. Nat Genet (2013). 45(10):1113-1120. doi:10.1038/ng.2764

14. Bakhoum MF, Esmaeli B. Molecular characteristics of uveal melanoma: insights from the cancer genome Atlas (TCGA) project. Cancers (2019). 11(8):1061. doi:10.3390/cancers11081061

15. He W, Chen L, Yuan K, Zhou Q, Peng L, Han Y. Gene set enrichment analysis and meta-analysis to identify six key genes regulating and controlling the prognosis of esophageal squamous cell carcinoma. J Thorac Dis (2018). 10(10): 5714-5726. doi:10.21037/jtd.2018.09.55

16. Subramanian A, Tamayo P, Mootha VK, Mukherjee S, Ebert BL, Gillette MA, et al. Gene set enrichment analysis: a knowledge-based approach for

\section{CONFLICT OF INTEREST}

The authors declare that the research was conducted in the absence of any commercial or financial relationships that could be construed as a potential conflict of interest.

\section{SUPPLEMENTARY MATERIAL}

The Supplementary Material for this article can be found online at: https://www.por-journal.com/articles/10.3389/pore.2021.596899/ full\#supplementary-material.

interpreting genome-wide expression profiles. Proc Natl Acad Sci (2005). 102(43):15545-15550. doi:10.1073/pnas.0506580102

17. Zhou Y, Zhou B, Pache L, Chang M, Khodabakhshi AH, Tanaseichuk O, et al. Metascape provides a biologist-oriented resource for the analysis of systems-level datasets. Nat Commun (2019). 10(1):1523. doi:10.1038/s41467-019-09234-6

18. Franz M, Rodriguez H, Lopes C, Zuberi K, Montojo J, Bader GD, et al. GeneMANIA update 2018. Nucleic Acids Res (2018). 46(W1):W60-W64. doi:10.1093/nar/gky311

19. Liu C-J, Hu F-F, Xia M-X, Han L, Zhang Q, Guo A-Y. GSCALite: a web server for gene set cancer analysis. Bioinformatics (2018). 34(21):3771-3772. doi:10. 1093/bioinformatics/bty411

20. Calses PC, Dhillon KK, Tucker N, Chi Y, Huang J-w., Kawasumi M, et al. DGCR8 mediates repair of UV-induced DNA damage independently of RNA processing. Cel Rep (2017). 19(1):162-174. doi:10.1016/j.celrep.2017.03.021

21. Torre LA, Bray F, Siegel RL, Ferlay J, Lortet-Tieulent J, Jemal A. Global cancer statistics, 2012. CA: A Cancer J Clinicians (2015). 65(2):87-108. doi:10.3322/ caac. 21262

22. Ma W, Zhang C-Q, Dang C-X, Cai H-Y, Li H-l., Miao G-Y, et al. Upregulated long-non-coding RNA DLEU2 exon 9 expression was an independent indicator of unfavorable overall survival in patients with esophageal adenocarcinoma. Biomed Pharmacother (2019). 113:108655. doi:10.1016/j. biopha.2019.108655

23. Dong G, Mao Q, Yu D, Zhang Y, Qiu M, Dong G, et al. Integrative analysis of copy number and transcriptional expression profiles in esophageal cancer to identify a novel driver gene for therapy. Sci Rep (2017). 7(1):42060. doi:10. 1038/srep42060

24. Zhu X, Luo X, Feng G, Huang H, He Y, Ma W, et al. CENPE expression is associated with its DNA methylation status in esophageal adenocarcinoma and independently predicts unfavorable overall survival. PLoS One (2019). 14(2): e0207341. doi:10.1371/journal.pone.0207341

25. Mao Y, Fu Z, Zhang Y, Dong L, Zhang Y, Zhang Q, et al. A seven-lncRNA signature predicts overall survival in esophageal squamous cell carcinoma. Sci Rep (2018). 8(1):8823. doi:10.1038/s41598-018-27307-2

26. Dong Z, Zhang H, Zhan T, Xu S. Integrated analysis of differentially expressed genes in esophageal squamous cell carcinoma using bioinformatics. Neoplasma (2018). 65(4):523-531. doi:10.4149/neo_2018_170708N470

27. Men CD, Liu QN, Ren Q. A prognostic 11 genes expression model for ovarian cancer. J Cel Biochem. (2018). 119(2):1971-1978. doi:10.1002/jcb.26358

28. Li J, Wang W, Xia P, Wan L, Zhang L, Yu L, et al. Identification of a fivelncRNA signature for predicting the risk of tumor recurrence in patients with breast cancer. Int J Cancer (2018). 143(9):2150-2160. doi:10.1002/ijc.31573

29. Chen L, Lu D, Sun K, Xu Y, Hu P, Li X, et al. Identification of biomarkers associated with diagnosis and prognosis of colorectal cancer patients based on integrated bioinformatics analysis. Gene (2019). 692:119-125. doi:10.1016/j. gene.2019.01.001

30. Sancar A, Lindsey-Boltz LA, Ünsal-Kaçmaz K, Linn S. Molecular mechanisms of mammalian DNA repair and the DNA damage checkpoints. Annu Rev Biochem (2004). 73:39-85. doi:10.1146/annurev.biochem.73.011303.073723

31. Rocha C, Silva M, Quinet A, Cabral-Neto J, Menck C. DNA repair pathways and cisplatin resistance: an intimate relationship. Clinics (2018). 73(Suppl. 1): e478s. doi:10.6061/clinics/2018/e478s 
32. Swahari V, Nakamura A, Baran-Gale J, Garcia I, Crowther AJ, Sons R, et al. Essential function of dicer in resolving DNA damage in the rapidly dividing cells of the developing and malignant cerebellum. Cel Rep (2016). 14(2): 216-224. doi:10.1016/j.celrep.2015.12.037

33. Wen J, Lv Z, Ding H, Fang X, Sun M. Association of miRNA biosynthesis genes DROSHA and DGCR8 polymorphisms with cancer susceptibility: a systematic review and meta-analysis. Biosci Rep (2018). 38(3):BSR20180072. doi:10.1042/ BSR20180072

34. Rodriguez-Bravo V, Pippa R, Song W-M, Carceles-Cordon M, DominguezAndres A, Fujiwara N, et al. Nuclear pores promote lethal prostate cancer by increasing pom121-driven E2F1, MYC, and AR nuclear import. Cell (2018). 174(5):1200-1215.e20. doi:10.1016/j.cell.2018.07.015

35. Guo J, Liu X, Wu C, Hu J, Peng K, Wu L, et al. The transmembrane nucleoporin Pom121 ensures efficient HIV-1 pre-integration complex nuclear import. Virology (2018). 521:169-174. doi:10.1016/j.virol.2018. 06.008

36. Saint M, Sawhney S, Sinha I, Singh RP, Dahiya R, Thakur A, et al. The TAF9 C-terminal conserved region domain is required for SAGA and TFIID promoter occupancy to promote transcriptional activation. Mol Cell Biol (2014). 34(9):1547-1563. doi:10.1128/mcb.01060-13

37. Yoon JW, Lamm M, Iannaccone S, Higashiyama N, Leong KF, Iannaccone P, et al. p53 modulates the activity of the GLIl oncogene through interactions with the shared coactivator TAF9. DNA Repair (2015). 34:9-17. doi:10.1016/j.dnarep.2015. 06.006

38. Huang L, Low A, Damle SS, Keenan MM, Kuntz S, Murray SF, et al. Antisense suppression of the nonsense mediated decay factor Upf3b as a potential treatment for diseases caused by nonsense mutations. Genome Biol (2018). 19(1):4. doi:10.1186/s13059-017-1386-9

39. Elsemman IE, Mardinoglu A, Shoaie S, Soliman TH, Nielsen J. Systems biology analysis of hepatitis $\mathrm{C}$ virus infection reveals the role of copy number increases in regions of chromosome $1 \mathrm{q}$ in hepatocellular carcinoma metabolism. Mol Biosyst (2016). 12(5):1496-1506. doi:10. 1039/c5mb00827a

40. Benevolenskaya EV, Islam ABMMK, Ahsan H, Kibriya MG, Jasmine F, Wolff $\mathrm{B}$, et al. DNA methylation and hormone receptor status in breast cancer. Clin Epigenet (2016). 8(1):17. doi:10.1186/s13148-016-0184-7

Copyright (C) 2021 Wang, Li, Zhao, Jiang, Song, Qi, Chen, Ju, Hu, Wei, He and Zhao. This is an open-access article distributed under the terms of the Creative Commons Attribution License (CC BY). The use, distribution or reproduction in other forums is permitted, provided the original author(s) and the copyright owner(s) are credited and that the original publication in this journal is cited, in accordance with accepted academic practice. No use, distribution or reproduction is permitted which does not comply with these terms. 